\title{
RECURRENCE FORMULAE FOR THE COEFFICIENTS OF MODULAR FORMS
}

\author{
TORLEIV KLøVE
}

1.

Let $\Gamma(1)$ denote the full modular group, that is, the group of matrices

$$
\left(\begin{array}{ll}
a & b \\
c & d
\end{array}\right), \quad a d-b c=1,
$$

where $a, b, c, d$ are integers, and let $\Gamma_{0}(m)$ denote the subgroup defined by $c \equiv 0(\bmod m)$. A multiplier system $v=v(\Gamma,-r)$ of dimension $-r$ for a group $\Gamma \subset \Gamma(1)$ is a complex valued function $v(M)$ defined on $M \in \Gamma$ such that

$$
\begin{gathered}
|v(M)|=1, \\
v(M N)\left(c_{M N} \tau+d_{M N}\right)^{r}=v(M)\left(c_{M} N \tau+d_{M}\right)^{r} v(N)\left(c_{N} \tau+d_{N}\right)^{r},
\end{gathered}
$$

for $M, N \in \Gamma$, where

$$
M=\left(\begin{array}{ll}
a_{M} & b_{M} \\
c_{M} & d_{M}
\end{array}\right) \quad \text { etc. }
$$

A modular form on $\Gamma$ of dimension $-r$ and multiplier system $v=v(\Gamma,-r)$ is a function $F(\tau)$, meromorphic in the fundamental domain $\Delta(\Gamma)$ of $\Gamma$, which satisfies

$$
F(M \tau)=v(M)\left(c_{M} \tau+d_{M}\right)^{r} F(\tau)
$$

for all $M \in \Gamma$ (see Lehner [3]). The set of all such modular forms is a vector space over the complex numbers, we denote it by $(\Gamma,-r, v)$. We denote by $C^{+}(\Gamma,-r, v)$ the subspace consisting of all regular forms, that is, modular forms which are regular in $\Delta(\Gamma)$. We denote by $C^{\circ}(\Gamma,-r, v)$ the subspace consisting of all cuspforms, that is, regular forms which are zero at the cusps of $\Delta(\Gamma)$.

In the case of integral dimension and multiplier system identically 1 , Hecke introduced an operator $T(n)$ which maps $C^{\circ}$ onto itself. He showed for some $C^{\circ}$ that they have a basis of forms which are simul-

Received March 6, 1969. 
taneous eigenforms for all $T(n)$. Petersson introduced a scalar product in $C^{\circ}$ and by means of it he proved that $C^{\circ}$ always has such a basis of forms.

The Hecke theory of modular forms of integral dimension was later studied extensively (for a summary see Rankin [6]). In principle it has been known for some time that there exists a Hecke theory for cuspforms of half integral dimension. The purpose of this paper is to work out the details of this theory for cuspforms of all multiplier systems on the full group $\Gamma(1)$. We define an operator $T\left(p^{2}\right)$ and show that this operator maps $C^{\circ}$ onto itself. Using this fact we show our Theorem 1 : The coefficients of every cuspform satisfy a recurrence relation with integral coefficients. Further we use Petersson's scalar product to prove our main result, Theorem 2: There exists a basis for $C^{\circ}\left(\Gamma(1),-\frac{1}{2} k, v\right)$, $k$ odd, such that all the basis forms are simultaneous eigenforms for all $T\left(p^{2}\right)$ with real algebraic integers as eigenvalues.

2.

Let

$$
\eta(\tau)=e\left(\frac{1}{24} \tau\right) \prod_{r=1}^{\infty}(1-e(r \tau)), \quad \operatorname{Im} \tau>0,
$$

where $e(t)=\exp (2 \pi i t)$. It is known that $\eta(\tau)$ is a cuspform on $\Gamma(1)$ of dimension $-\frac{1}{2}$. Newman [5] and van Lint [4] have found recurrence relations for the coefficients of $\eta(\tau)^{k}, 0<k<24$. Newman [5] proved the existence of recurrence relations for $\eta(\tau)^{k}$ for all $k>0$.

The multiplier system $v_{0}$ of $\eta(\tau)$ is given by (2.1)-(2.4) below (see Weber [7]). For $\operatorname{Im} \tau>0$ we have

$$
v_{0}\left(\left(\begin{array}{ll}
a & b \\
c & d
\end{array}\right)\right)=\left(\frac{d}{c}\right) e\left(\frac{1}{24}\left(c(a+d)+b d\left(1-c^{2}\right)-3 c\right)\right)
$$

when $c$ is positive and odd, $d \neq 0$, where $\operatorname{Im}(c \tau+d)^{\frac{1}{2}}>0$;

$$
v_{0}\left(\left(\begin{array}{ll}
a & b \\
c & d
\end{array}\right)\right)=\left(\frac{c}{d}\right) e\left(\frac{1}{24}\left(d(b-c)+a c\left(1-d^{2}\right)+3 d-3\right)\right)
$$

when $d$ is positive and odd, $c \neq 0$, where $\operatorname{Re}(c \tau+d)^{\frac{1}{2}}>0$;

$$
\begin{aligned}
& v_{0}\left(\left(\begin{array}{rr}
0 & -1 \\
1 & 0
\end{array}\right)\right)=e\left(-\frac{1}{8}\right), \quad \text { where } \operatorname{Im} \tau^{\frac{1}{2}}>0 ; \\
& v_{0}\left(\left(\begin{array}{ll}
1 & b \\
0 & 1
\end{array}\right)\right)=e\left(\frac{1}{24} b\right) ;
\end{aligned}
$$


where $\left(\frac{d}{c}\right)$ etc. are Jacobi's symbol. We also add a general formula valid for all multiplier systems $v=v(\Gamma,-r)$,

$$
v\left(\left(\begin{array}{rr}
-1 & 0 \\
0 & -1
\end{array}\right)\right)=e\left(\frac{1}{2} r\right) .
$$

Throughout this paper $p$ and $q$ denote odd primes, $k$ an odd integer $>0$. The group $\Gamma_{0}\left(p^{2}\right)$ is a subgroup of $\Gamma(1)$ of index $p(p+1)$. A set of right coset representatives for $\Gamma_{0}\left(p^{2}\right)$ in $\Gamma(1)$ is given by

$$
\left\{R^{\alpha}\left(\alpha=0,1, \ldots, p^{2}-1\right), R^{\beta p} T(\beta=0,1, \ldots, p-1)\right\},
$$

where

$$
\begin{gathered}
R=\left(\begin{array}{rr}
1 & 0 \\
-1 & 1
\end{array}\right), \quad T=\left(\begin{array}{rr}
0 & -1 \\
1 & 0
\end{array}\right) \\
R^{\alpha}=\left(\begin{array}{rr}
1 & 0 \\
-\alpha & 1
\end{array}\right), \quad R^{\beta p} T=\left(\begin{array}{rr}
0 & -1 \\
1 & \beta p
\end{array}\right) .
\end{gathered}
$$

Let $f(\tau) \in C^{\circ}\left(\Gamma(1),-\frac{1}{2} k, v\right)$. Define $T\left(p^{2}\right)$ by

$$
f(\tau) \mid T\left(p^{2}\right)=p^{k} \sum_{i} \frac{f\left(P R_{i} \tau\right)}{v\left(R_{i}\right)\left(c_{i} \tau+d_{i}\right)^{\frac{1}{2} k}}
$$

where

$$
P=\left(\begin{array}{cc}
p^{2} & 0 \\
0 & 1
\end{array}\right), \quad R_{i}=\left(\begin{array}{cc}
a_{i} & b_{i} \\
c_{i} & d_{i}
\end{array}\right)
$$

and $R_{i}$ runs through the set (2.6) of right coset representatives.

We use the notation

$$
\begin{aligned}
\delta(x) & =1 & & \text { if } x \text { is integral } \\
& =0 & & \text { otherwise }
\end{aligned}
$$

We shall prove

Theorem 1. Let $\mu$ be the dimension of $C^{\circ}=C^{\circ}\left(\Gamma(1),-\frac{1}{2} k, v_{0}{ }^{\alpha}\right)$, where $\varkappa \equiv k(\bmod 4), 0<\varkappa<24$, and $v_{0}$ is the multiplier system of $\eta(\tau)$. Further let

$$
f(\tau)=\Sigma_{n} A(n) e\left(\frac{1}{24}(k, 24) n \tau\right)
$$

be any form in $C^{\circ}$. If $k\left(p^{2}-1\right) \equiv \varkappa\left(p^{2}-1\right) \equiv 0(\bmod 24)$, then there exist integers $\Delta, \Delta_{1}, \ldots, \Delta_{\mu}$, not all zero, depending on $C^{\circ}$ and $p$ only, such that $\Delta_{\mu}=1$ and 


$$
\sum_{\alpha=1}^{\mu} \Delta_{\alpha}\{\ldots\}=\Delta(A(n))
$$

where

and

$$
\begin{aligned}
\{\ldots\}= & p^{2 \alpha} A\left(p^{2 \alpha} n\right)+p^{k \alpha} A\left(p^{-2 \alpha} n\right)+ \\
& +\sum_{u=1}^{\alpha} p^{\sharp((2 \alpha-2 u+1) k+4 u-3)} \gamma\left(\frac{\delta\left(n p^{2 u-2 \alpha}\right) n p^{2 u-2 \alpha}}{p}\right) A\left(p^{4 u-2 \alpha-2} n\right)+ \\
& +\sum_{u=1}^{\alpha-1} p^{(\alpha-u) k}\left(p^{2 u} \delta\left(n p^{2 u-2 \alpha}\right)-p^{2 u-1} \delta\left(n p^{2 u-2 \alpha+1}\right)\right) A\left(p^{4 u-2 \alpha n}\right)
\end{aligned}
$$

$$
\gamma=(-1)^{\frac{1}{1}(p-1)(x-1)}\left(\frac{3 /(k, 3)}{p}\right) .
$$

Theorem 2. Let $C^{\circ}$ be the same vector space as in Theorem 1. Then there exists a basis

$$
\left\{f_{v}(\tau) ; \nu=1,2, \ldots, \mu\right\}
$$

for $C^{\circ}$ such that all $f_{v}(\tau)$ are eigenforms with real eigenvalues for all $T\left(p^{2}\right)$, $p>3$, and for $T\left(3^{2}\right)$ if $k \equiv 0(\bmod 3)$ and $\varkappa \equiv k(\bmod 12)$. That is, there exist real algebraic integers $\varrho(v, p)$ such that

$$
f_{v}(\tau) \mid T\left(p^{2}\right)=\varrho(\nu, p) f_{\nu}(\tau) .
$$

Corollary. If

then

$$
f_{\nu}(\tau)=\Sigma_{n} A_{v}(n) e\left(\frac{1}{24}(k, 24) n \tau\right),
$$

$$
\begin{aligned}
p^{2} A_{\nu}\left(p^{2} n\right)+p^{\ddagger(k+1)}(-1)^{\frac{1}{4}(p-1)(x-1)}\left(\frac{3 n /(k, 3)}{p}\right) A_{\nu}(n)+p^{k} A_{v}\left(p^{-2} n\right) \\
=\varrho(v, p) A_{v}(n) .
\end{aligned}
$$

For the value of $\mu$, see Lemma 1 below.

3.

In this section we shall prove Theorem 1.

Let $\left.v\left(\Gamma(1),-\frac{1}{2} k\right)\right)=v_{0}$ ' be a multiplier system, $v_{0}$ are given by (2.1)(2.4). Let

Then

$$
U=\left(\begin{array}{ll}
1 & 1 \\
0 & 1
\end{array}\right)
$$

$$
v(U)=e\left(\frac{1}{24} \varkappa\right) \text {. }
$$

Hence (see Lehner [3, p. 347])

$$
6 \cdot \frac{1}{24} x-\frac{1}{4} k
$$

is an integer, and we have $\varkappa \equiv k(\bmod 4)$, which gives 6 possible values 
of $x, 0<x<24$. In the following we always assume $x \equiv k(\bmod 4)$ and $0<x<24$. All these values of $\varkappa$ give modular forms, e.g.

where

$$
\begin{aligned}
& \eta^{k}, \quad Q^{2} R \eta^{k-28}, \quad Q \eta^{k-8}, \\
& R \eta^{k-12}, \quad Q^{2} \eta^{k-16}, \quad Q R \eta^{k-20}
\end{aligned}
$$

Let

$$
\begin{aligned}
& Q(\tau)=1+240 \sum_{n=1}^{\infty} \sigma_{3}(n) e(n \tau), \\
& R(\tau)=1-504 \sum_{n=1}^{\infty} \sigma_{5}(n) e(n \tau), \\
& \sigma_{s}(n)=\sum_{d \mid n} d^{s} .
\end{aligned}
$$

$$
f(\tau) \in C^{\circ}\left(\Gamma(1),-\frac{1}{2} k, v_{0}{ }^{\varkappa}\right),
$$

where $\varkappa \equiv k(\bmod 24)$. Then

$$
f(\tau) \eta^{-k}(\tau) \in(\Gamma(1), 0,1),
$$

and it is regular inside $D=\Delta(\Gamma(1)$ ). At $\tau=i \infty$ (which we may choose as the only cusp of $D$ ) the local uniformizing variable is $x=e(\tau)$. Hence $f(\tau) \eta^{-k}(\tau)$ is regular or has a pole of order $<\frac{1}{24} k$ at $\tau=i \infty$. Therefore,

$$
f(\tau) \eta^{-k}(\tau)=\operatorname{Pol}_{\alpha}(j(\tau)),
$$

a polynomial of degree $\alpha \leqq\left[\frac{1}{24}(k-1)\right]$ in $j(\tau)$, where

$$
j(\tau)=12^{3} Q^{3} /\left(Q^{3}-R^{2}\right)
$$

is Klein's Hauptmodul of $\Gamma(1)$. This yields

Hence

$$
f(\tau)=\eta^{k}(\tau) \operatorname{Pol}_{\alpha}(j(\tau)) .
$$

$$
\eta^{k}(\tau) j^{\beta}(\tau), \quad \beta=0,1, \ldots,\left[\frac{1}{24}(k-1)\right],
$$

span $C^{\circ}\left(\Gamma(1),-\frac{1}{2} k, v_{0}{ }^{x}\right)$. Since they are linearly independent, $C^{\circ}$ has dimension

$$
\mu=\left[\frac{1}{24}(k-1)\right]+1 \text {. }
$$

Treating the other cases similarly we obtain

Lemma 1. Let $\varkappa \equiv k-a(\bmod 24)$. Let $\mu=\mu(k, a)$ be the dimension of $C^{\circ}\left(\Gamma(1),-\frac{1}{2} k, v_{0}{ }^{x}\right)$. Then

and

$$
\mu(k, a)=\left[\frac{1}{24}(k-a-1)\right]+1 \text { if } a \neq 4,
$$




$$
\begin{aligned}
\mu(k, 4) & =\left[\frac{1}{24}(k-5)\right] & & \text { if } k>3, \\
& =0 & & \text { if } k=1 \text { or } k=3 .
\end{aligned}
$$

Corollary. The dimension $\mu$ is finite.

Let $f(\tau) \in(\Gamma, 0,1)$ where $\Gamma \subset \Gamma(1)$ and let $M=\left\{R_{i}\right\}$ be a set of right coset representatives for $\Gamma$ in $\Gamma(1)$. If $V \in \Gamma(1)$, then $R_{i} V=G R_{j}$, where $G \in \Gamma$ and $R_{j} \in M$, and $R_{j}$ runs through $M$ when $R_{i}$ does so. Hence we get

LEMma 2. If $f(\tau) \in(\Gamma, 0,1)$ where $\Gamma$ is a subgroup of $\Gamma(1)$, then

$$
\Sigma_{i} f\left(R_{i} \tau\right) \in(\Gamma(1), 0,1),
$$

where the sum is over a set of right coset representatives for $\Gamma$ in $\Gamma(1)$.

Let $l=\left(24, p^{2}-1\right)$. Then $l=24$ when $p>3$, and $l=8$ when $p=3$. Applying (2.1)-(2.4) we obtain

Lemma 3. If $f(\tau) \in\left(\Gamma(1),-\frac{1}{2} k, v_{0}{ }^{x}\right)$ and $k \equiv \varkappa \equiv 0(\bmod 24 / l)$, then

$$
f(P \tau) f^{-1}(\tau) \in\left(\Gamma_{0}\left(p^{2}\right), 0,1\right) .
$$

Combining Lemmas 2 and 3 we obtain

This yields

$$
\sum_{i} \frac{f\left(P R_{i} \tau\right)}{v\left(R_{i}\right)\left(c_{i} \tau+d_{i}\right)^{\frac{1}{k} k} f(\tau)} \in(\Gamma(1), 0,1)
$$

Lemma 4. If $f(\tau) \in\left(\Gamma(1),-\frac{1}{2} k, v_{0}{ }^{x}\right)$ and $k \equiv \varkappa \equiv 0(\bmod 24 / l)$, then

$$
f(\tau) \mid T\left(p^{2}\right) \in\left(\Gamma(1),-\frac{1}{2} k, v_{0}{ }^{x}\right) .
$$

From (2.8) we obtain

$$
\begin{gathered}
P R^{\beta p} T=\left(\begin{array}{cc}
0 & -p^{2} \\
1 & \beta p
\end{array}\right)=\left(\begin{array}{cc}
0 & -1 \\
1 & (\beta-l \delta) / p
\end{array}\right)\left(\begin{array}{ll}
1 & l p \delta \\
0 & p^{2}
\end{array}\right), \\
P R^{\alpha}=\left(\begin{array}{cc}
p^{2} & 0 \\
-\alpha & 1
\end{array}\right)=\left(\begin{array}{cc}
p^{2} & -l \delta \\
-\alpha & (1+\alpha l \delta) / p^{2}
\end{array}\right)\left(\begin{array}{ll}
1 & l \delta \\
0 & p^{2}
\end{array}\right), \\
P R^{\alpha p}=\left(\begin{array}{cc}
p^{2} & 0 \\
-\alpha p & 1
\end{array}\right)=\left(\begin{array}{cc}
p & -l \delta \\
-\alpha & (1+\alpha l \delta) / p
\end{array}\right)\left(\begin{array}{ll}
p & l \delta \\
0 & p
\end{array}\right) .
\end{gathered}
$$

Let

$$
D_{1}=(\beta-l \delta) / p,
$$

where $\beta-l \delta \equiv 0(\bmod p), 0 \leqq \delta<p$; 


$$
D_{2}=(1+\alpha l \delta) / p^{2}
$$

where $p \nmid \alpha$ and $1+\alpha l \delta \equiv 0\left(\bmod p^{2}\right), 0<\delta<p^{2}$;

$$
D_{3}=(1+\alpha l \delta) / p \text {, }
$$

where $p \nmid \alpha$ and $1+\alpha l \delta \equiv 0(\bmod p), 0<\delta<p$. Then

Hence

$$
D_{1} \equiv p^{2} D_{1} \equiv p \beta(\bmod l)
$$

Similarly

$$
x D_{1} \equiv x p \beta(\bmod 24)
$$

$$
x D_{2} \equiv \varkappa(\bmod 24), \quad x D_{3} \equiv \varkappa p(\bmod 24)
$$

Putting $n=\varepsilon 2^{\gamma} n_{1}$, where $\varepsilon= \pm 1, \gamma \geqq 0, n_{1}>0$, and $n_{1}$ odd, we obtain

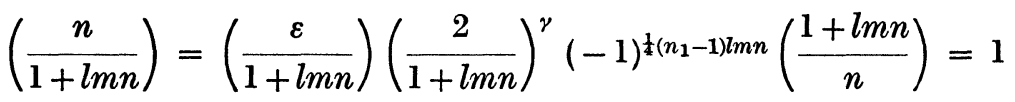

since $8 \mid l$. Now, from (3.1)-(3.3) and the remarks we have made, we obtain, by (2.1)-(2.4),

$$
\begin{aligned}
\frac{f\left(P R^{\beta p} T \tau\right)}{v\left(R^{\beta p} T\right)(c \tau+d)^{\frac{1}{2} k}} & =\frac{\left(\frac{D_{1}}{1}\right) e\left(\frac{1}{24} \varkappa\left(D_{1}-3\right)\right)\left(\frac{\tau+l p \delta}{p^{2}}+D_{1}\right)^{\frac{1}{2} k} f\left(\frac{\tau+l p \delta}{p^{2}}\right)}{\left(\frac{\beta p}{1}\right) e\left(\frac{1}{24} \varkappa(p \beta-3)\right)(\tau+\beta p)^{\frac{1}{2} k}} \\
& =p^{-k} f\left(\frac{\tau+l p \delta}{p^{2}}\right) .
\end{aligned}
$$

If $p \nmid \alpha$, then

$$
\frac{f\left(P R^{\alpha} \tau\right)}{v\left(R^{\alpha}\right)(c \tau+d)^{\frac{1}{2} k}}
$$

$=\frac{\left(\frac{-\alpha}{D_{2}}\right) e\left(\frac{1}{24} \varkappa\left(D_{2}(\alpha-l \delta)-\alpha p^{2}\left(1-D_{2}^{2}\right)+3\left(D_{2}-1\right)\right)\right)\left(-\alpha \frac{\tau+l \delta}{p^{2}}+D_{2}\right)^{\frac{1}{2} k} f\left(\frac{\tau+l \delta}{p^{2}}\right)}{\left(\frac{-\alpha}{1}\right) e\left(\frac{1}{24} \varkappa \alpha\right)(-\alpha \tau+1)^{\frac{1}{2} k}}$

$=p^{-k} f\left(\frac{\tau+l \delta}{p^{2}}\right)$

since

$$
\left(\frac{-\alpha}{D_{2}}\right)=\left(\frac{-\alpha}{p^{2} D_{2}}\right)=\left(\frac{-\alpha}{1+\alpha l \delta}\right)=1 \text { and } x\left(D_{2}-1\right) \equiv 0(\bmod 24) .
$$


If $p \nmid \alpha$, then

$$
\begin{aligned}
& \frac{f\left(P R^{\alpha p} \tau\right)}{v\left(R^{\alpha p}\right)(c \tau+d)^{\frac{1}{1} k}} \\
&=\frac{\left(\frac{-\alpha}{D_{3}}\right) e\left(\frac{1}{24} \varkappa\left(D_{3}(\alpha-l \delta)-p \alpha\left(1-D_{3}{ }^{2}\right)+3 D_{3}-3\right)\right)\left(-\alpha \frac{p \tau+l \delta}{p}+D_{3}\right)^{\frac{1}{2} k} f\left(\frac{p \tau+l \delta}{p}\right)}{\left(\frac{-\alpha p}{1}\right) e\left(\frac{1}{24} \varkappa \alpha p\right)(-\alpha p \tau+1)^{\frac{1}{2} k}}
\end{aligned}
$$$$
=\left(\frac{-\alpha}{D_{3}}\right) p^{-\frac{1}{2} k} e\left(\frac{1}{24} \varkappa \cdot 3(p-1)\right) f\left(\frac{p \tau+l \delta}{p}\right) \text {. }
$$

Since $-\alpha l \delta \equiv 1(\bmod p)$, we have

$$
\left(\frac{-\alpha}{D_{3}}\right)=\left(\frac{-\alpha}{p^{2} D_{3}}\right)=\left(\frac{-\alpha}{p}\right)\left(\frac{-\alpha}{1+\alpha l \delta}\right)=\left(\frac{-\alpha}{p}\right)=\left(\frac{l \delta}{p}\right) .
$$

Hence we obtain

LeMma 5. If $f(\tau) \in\left(\Gamma(1),-\frac{1}{2} k, v_{0}{ }^{x}\right)$ and $k \equiv \varkappa \equiv 0(\bmod 24 / l)$, then

$$
\begin{aligned}
f(\tau) \mid T\left(p^{2}\right)= & \sum_{\delta=0}^{p 2-1} f\left(\frac{\tau+l \delta}{p^{2}}\right)+p^{k} f\left(p^{2} \tau\right)+ \\
& +p^{\frac{1}{3} k} \sum_{\delta=1}^{p-1}\left(\frac{l \delta}{p}\right) e\left(\frac{1}{8} \varkappa(p-1)\right) f\left(\frac{p \tau+l \delta}{p}\right) .
\end{aligned}
$$

In [4] van Lint considered the case $\varkappa \equiv k(\bmod 24)$. He defined $T\left(p^{2}\right)$ differently; however, he obtained the form given in Lemma 5.

From Lemma 5 we obtain (as in Kløve [2])

LEMMA 6. If $k \equiv \varkappa \equiv 0(\bmod 24 / l)$,

and

$$
f(\tau)=\Sigma_{n} A(n) e\left(\frac{1}{24}(k, 24) n \tau\right) \in\left(\Gamma(1),-\frac{1}{2} k, v_{0}{ }^{x}\right),
$$

then

$$
f(\tau) \mid T\left(p^{2}\right)=\Sigma_{n} A^{*}(n) e\left(\frac{1}{24}(k, 24) n \tau\right)
$$

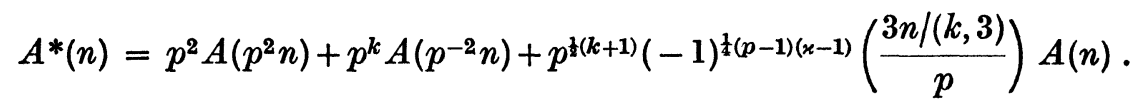

Lemmas 5 and 6 yields

LEMMa 7. If $k \equiv \varkappa \equiv 0(\bmod 24 / l)$ and

$$
f(\tau) \in C^{\circ}\left(\Gamma(1),-\frac{1}{2} k, v_{0}{ }^{\alpha}\right),
$$


then

$$
f(\tau) \mid T\left(p^{2}\right) \in C^{\circ}\left(\Gamma(1),-\frac{1}{2} k, v_{0}^{x}\right)
$$

For convenience we write $f(\tau)|T(m)| T(n)=f(\tau) \mid T(m) T(n)$ in the following. As in van Lint [4] we may define $T\left(p^{2 \alpha}\right)$ by

This yields

$$
\begin{aligned}
f(\tau) \mid T\left(p^{2 \alpha}\right)= & \sum_{b=0}^{2 \alpha_{-1}} f\left(\frac{\tau+l b}{p^{2 \alpha}}\right)+p^{k \alpha} f\left(p^{2 \alpha} \tau\right)+ \\
& +\sum_{v=1}^{2 \alpha-1} \sum_{b=0}^{p^{v-1}} e\left(\frac{1}{8} \varkappa\left(p^{v}-1\right)\right)\left(\frac{l b}{p}\right)^{v} p^{\frac{1}{2} k(2 \alpha-v)} f\left(\frac{p^{2 \alpha-v} \tau+l b}{p^{v}}\right) .
\end{aligned}
$$

$$
\begin{aligned}
& T\left(p^{4}\right)=T\left(p^{2}\right) T\left(p^{2}\right)-p^{k+1}(p+1), \\
& T\left(p^{2 \alpha}\right)=T\left(p^{2}\right) T\left(p^{2 \alpha-2}\right)-p^{k+2} T\left(p^{2 \alpha-4}\right) \quad \text { when } \alpha>2 .
\end{aligned}
$$

For $k-x \equiv 0,4,8,12,16,20(\bmod 24)$, respectively, let $A=1, Q^{2} R, Q$, $R, Q^{2}, Q R$. Then

$$
\left\{f_{\beta}=A \eta^{24(\mu-1)+x} j^{\beta} ; \beta=0,1, \ldots, \mu-1\right\}
$$

is a basis for $C^{\circ}\left(\Gamma(1),-\frac{1}{2} k, v_{0}{ }^{\varkappa}\right)$. When expanded in powers of $e\left(\frac{1}{24} \tau\right)$, each of the forms in (3.5) has integral Fourier coefficients of which the first non-zero equals 1. Using the basis (3.5) of $C^{\circ}$ we associate with $T\left(p^{2}\right)$ a matrix $H=H(p)$ such that

$$
\left(\sum_{\beta=0}^{\mu-1} a_{\beta} f_{\beta}\right) H=\left(\sum_{\beta=0}^{\mu-1} a_{\beta} f_{\beta}\right) \mid T\left(p^{2}\right) .
$$

It is clear that $H$ has integral elements. Let

$$
\sum_{l=0}^{\mu} b_{l} \varrho^{l} ; \quad b_{\mu}=(-1)^{\mu},
$$

be the characteristic polynomial of $H$. Then all $b_{l}$ are integers since $H$ has integral elements. By Cayley-Hamilton's theorem we get

$$
f \mid \sum_{l=0}^{\mu} b_{l} T\left(p^{2}\right)^{l}=0
$$

for all $f$ in $C^{\circ}$. Hence, by (3.4)

$$
f \mid \sum_{\alpha=0}^{\mu} \Delta_{\alpha} T\left(p^{2 \alpha}\right)=0
$$

where $\Delta_{\mu}=1$ and all $\Delta_{\alpha}$ are integers. Translating to terms of the Fourier coefficients $A(n)$ we obtain Theorem 1 as in Kløve [2]. There we used modular functions rather than forms, and we treated only the case $f(\tau)=\eta^{k}(\tau)$; the general case, however, is quite similar.

Further, the eigenvalues of $T\left(p^{2}\right)$ are algebraic integers by (3.6). 
4.

If $p \neq q$, then Lemma 6 yields

where

$$
f(\tau) \mid T\left(p^{2}\right) T\left(q^{2}\right)=\Sigma_{n} B(n) e\left(\frac{1}{24}(k, 24) n \tau\right),
$$

$$
\begin{aligned}
& B(n)=(p q)^{2} A\left(p^{2} q^{2} n\right)+(p q)^{k} A\left(p^{-2} q^{-2} n\right)+ \\
& +p^{k} q^{2} A\left(p^{-2} q^{2} n\right)+q^{k} p^{2} A\left(q^{-2} p^{2} n\right)+ \\
& +p^{\frac{1}{2}(k+1)}(-1)^{\frac{1}{(}(p-1)(x-1)}\left(\frac{3 n /(k, 3)}{p}\right)\left(q^{2} A\left(q^{2} n\right)+q^{k} A\left(q^{-2} n\right)\right)+ \\
& +q^{\frac{1}{2}(k+1)}(-1)^{\frac{1}{(q-1)(x-1)}}\left(\frac{3 n /(k, 3)}{q}\right)\left(p^{2} A\left(p^{2} n\right)+p^{k} A\left(p^{-2} n\right)\right)+ \\
& +(p q)^{\frac{1}{2}(k+1)}(-1)^{\frac{1}{1}(p+q-2)(x-1)}\left(\frac{3 n /(k, 3)}{p q}\right) A(n) .
\end{aligned}
$$

This is symmetric in $p$ and $q$. Hence

Lemma 8. We have

$$
f(\tau)\left|T\left(p^{2}\right) T\left(q^{2}\right)=f(\tau)\right| T\left(q^{2}\right) T\left(p^{2}\right) .
$$

Petersson defines

$$
(f, g)=\iint_{\Delta(\Gamma)} f(\tau) \overline{g(\tau)} y^{r-2} d x d y,
$$

where $\tau=x+i y, f(\tau), g(\tau) \in C^{+}(\Gamma,-r, v)$ and at least one of $f$ and $g$ belongs to $C^{\circ}(\Gamma,-r, v) ; \Gamma$ is a subgroup of $\Gamma(1)$ and $\Delta=\Delta(\Gamma)$ is a fundamental domain for $\Gamma$. The integral is independent of the choice of a fundamental domain, and it converges under the conditions stated.

Again, let $\Gamma$ be a subgroup of $\Gamma(1),\left\{R_{i}\right\}$ a set of right coset representatives for $\Gamma$ in $\Gamma(1)$, and $\Delta$ a fundamental domain for $\Gamma(1)$. Let $\tau$ be in the upper half plane. Then $\tau=( \pm M) \tau_{0}$ where $\tau_{0} \in \Delta$ and $M \in \Gamma(1)$, and the representation is unique. Further $M=N R_{i}$ where $N \in \Gamma$, again the representation is unique. Hence $\tau=( \pm N) R_{i} \tau_{0}$, and we obtain

Lemma 9. Let $\Delta$ be a fundamental domain for $\Gamma(1)$ and $\left\{R_{i}\right\}$ a set of right coset representatives for $\Gamma$ in $\Gamma(1)$ where $\Gamma$ is a subgroup of $\Gamma(1)$. Then

is a fundamental domain for $\Gamma$.

$$
\cup_{i} R_{i} \Delta
$$


Let $f(\tau), g(\tau) \in C^{\circ}\left(\Gamma(1),-\frac{1}{2} k, v_{0}{ }^{x}\right)=C^{\circ}(\Gamma(1),-r, v)$. Then

$$
\left(f(\tau) \mid T\left(p^{2}\right), g(\tau)\right)=p^{k} \iint_{\Delta(T(1))} \sum_{i} \frac{f\left(P R_{i} \tau\right)}{v\left(R_{i}\right)\left(c_{i} \tau+d_{i}\right)^{r}} \overline{g(\tau)} y^{r-2} d x d y .
$$

Let $\tau^{\prime}=R_{i} \tau=x^{\prime}+i y^{\prime}$, where

Then

$$
R_{i}=\left(\begin{array}{ll}
a_{i} & b_{i} \\
c_{i} & d_{i}
\end{array}\right), \quad R_{i}^{-1}=\left(\begin{array}{ll}
a_{-i} & b_{-i} \\
c_{-i} & d_{-i}
\end{array}\right) .
$$

$$
y^{\prime}=y\left|c_{i} \tau+d_{i}\right|^{-2}, \quad\left(y^{\prime}\right)^{-2} d x^{\prime} d y^{\prime}=y^{-2} d x d y .
$$

Further (1.1) yields

(1.2) yields

$$
v\left(R_{i}\right)^{-1}=\overline{v\left(R_{i}\right)},
$$

$$
v\left(R_{i}^{-1}\right)\left(c_{-i} \tau^{\prime}+d_{-i}\right)^{r} v\left(R_{i}\right)\left(c_{i} \tau+d_{i}\right)^{r}=1,
$$

and (1.3) yields

Hence

$$
g(\tau)=g\left(R_{i}^{-1} \tau^{\prime}\right)=v\left(R_{i}^{-1}\right)\left(c_{-i} \tau^{\prime}+d_{-i}\right)^{r} g\left(\tau^{\prime}\right) .
$$

$$
\begin{aligned}
& \frac{f\left(P R_{i} \tau\right)}{\overline{v\left(R_{i}\right)\left(c_{i} \tau+d_{i}\right)^{r}}} \overline{g(\tau)} y^{r-2} d x d y \\
& \quad=\frac{\overline{v\left(R_{i}\right)} f\left(P \tau^{\prime}\right)}{\left(c_{i} \tau+d_{i}\right)^{r}} \overline{v\left(R_{i}^{-1}\right)\left(c_{-i} \tau^{\prime}+d_{-i}\right)^{r} g\left(\tau^{\prime}\right)}\left|c_{i} \tau+d_{i}\right|^{2 r} y^{\prime r-2} d x^{\prime} d y^{\prime} \\
& \quad=f\left(P \tau^{\prime}\right) \overline{g\left(\tau^{\prime}\right)} y^{\prime r-2} d x^{\prime} d y^{\prime} .
\end{aligned}
$$

This yields

$$
\left(f(\tau) \mid T\left(p^{2}\right), g(\tau)\right)=p^{k} \sum_{i} \int_{R_{i} \triangleleft(\Gamma(1))} f(P \tau) \overline{g(\tau)} y^{r-2} d x d y .
$$

Hence, by Lemma 9 we obtain

Lemma 10. If $k \equiv \varkappa \equiv 0(\bmod 24 / l)$ and

then

$$
f(\tau), g(\tau) \in C^{\circ}\left(\Gamma(1),-\frac{1}{2} k, v_{0}{ }^{\varkappa}\right),
$$

$$
\left(f(\tau) \mid T\left(p^{2}\right), g(\tau)\right)=p^{k} \iint_{\Delta\left(\Gamma_{0}\left(p^{2}\right)\right)} f(P \tau) \overline{g(\tau)} y^{r-2} d x d y .
$$

Let

$$
W=\left(\begin{array}{cr}
0 & -1 \\
p^{2} & 0
\end{array}\right)
$$


$W$ is in the normalizer for $\Gamma_{0}\left(p^{2}\right)$. Hence, if $\Delta$ is a fundamental domain for $\Gamma_{0}\left(p^{2}\right)$, then so is $W \Delta$. Let $\tau^{\prime}=W \tau$. Then

$$
\begin{aligned}
f\left(\tau^{\prime}\right) \overline{g\left(P \tau^{\prime}\right)} y^{\prime r-2} d x^{\prime} d y^{\prime} & =f\left(-p^{-2} \tau^{-1}\right) \overline{g\left(-\tau^{-1}\right)} y^{r-2} p^{-2 r}|\tau|^{-2 r} d x d y \\
& =v(T)\left(p^{2} \tau\right)^{r} f\left(p^{2} \tau\right) \overline{v(T)} \overline{\tau^{r}} \overline{g(\tau)} y^{r-2} p^{-2 r}|\tau|^{-2 r} d x d y \\
& =f(P \tau) \overline{g(\tau)} y^{r-2} d x d y .
\end{aligned}
$$

Hence

$$
\begin{aligned}
&\left(f(\tau) \mid T\left(p^{2}\right), g(\tau)\right)=p^{k} \iint_{W \Delta\left(\Gamma_{0}\left(p^{2}\right)\right)} f(\tau) \overline{g(P \tau)} y^{r-2} d x d y \\
&=p^{k} \int_{\Delta\left(\Gamma_{0}\left(p^{2}\right)\right)} g(P \tau) \overline{f(\tau)} y^{r-2} d x d y \\
& i=\overline{\left(g(\tau) \mid T\left(p^{2}\right), f(\tau)\right)=}\left(f(\tau), g(\tau) \mid T\left(p^{2}\right)\right) .
\end{aligned}
$$

LEMMA 11. If $k \equiv \varkappa \equiv 0(\bmod 24 / l)$ and

then

$$
f(\tau), g(\tau) \in C^{\circ}\left(\Gamma(1),-\frac{1}{2} k, v_{0}{ }^{\varkappa}\right),
$$

$$
\left(f(\tau) \mid T\left(p^{2}\right), g(\tau)\right)=\left(f(\tau), g(\tau) \mid T\left(p^{2}\right)\right) \text {. }
$$

Theorem 2 now follows from Lemmas 1, 7, 8 and 11 (see e.g. Gunning [1]). The corollary follows from Theorem 2 and Lemma 6.

\section{REFERENCES}

1. R. C. Gunning, Lectures on modular forms (Annals of Math. Studies 48), Princeton, 1962.

2. T. Kløve, Recurrence formulae for the coefficients of modular forms and congruences for the partition function and for the coefficients of $j(\tau),(j(\tau)-1728)^{\frac{1}{2}}$, and $j(\tau)^{\frac{1}{3}}$, Math. Scand. 23 (1968), 133-159.

3. J. Lehner, Discontinuous groups and automorphic functions (Math. Surveys 8), Amer. Math. Soc., Providence, R.I., 1964.

4. J. H. van Lint, Hecke operators and Euler products, Druckerij "Luctor et Emergo", Leiden, 1957.

5. M. Newman, On the existence of identities for the coefficients of certain modular forms, J. London Math. Soc. 31 (1956), 350-359.

6. R. A. Rankin, Hecke operators on congruence subgroups of the modular group, Math. Ann. 168 (1967), 40-58.

7. H. Weber, Elliptische Functionen und algebraische Zahlen, Braunschweig, 1891. 